CUBO A Mathematical Journal Vol.13, No으, (73-84). June 2011

\title{
Closure of Pointed Cones and Maximum Principle in Hilbert Spaces
}

\author{
Paolo D'Alessandro \\ Math. Department, \\ Third University of Rome, \\ email: dalex@mat.uniroma3.it
}

\begin{abstract}
We prove, in a Hilbert space setting, that all targets of the minimum norm optimal control problems reachable with inputs of minimum norm $\rho$ are support points for the the set reachable by inputs with norm bounded by $\rho$. This amount to say that the Maximum Principle always holds in Hilbert Spaces.
\end{abstract}

\section{RESUMEN}

En este artículo se demuestra que, para el problema de control óptimo a un nivel mínimo en los espacios de Hilbert, todos los estados alcanzables con un nivel mínimo de entrada de $\rho$ son puntos de apoyo para el conjunto de estados alcanzables por la norma de entrada inferior o igual a $\rho$. Esto es equivalente a decir que el Principio Máximo siempre es válido en los espacios de Hilbert.

Keywords and phrases: Linear Control Systems in Hilbert Spaces, Norm Optimal Control, Maximum Principle.

Mathematics Subject Classification: 93E20, 93E25. 


\section{Introduction}

Arguing in a Hilbert space setting, suppose that the minimum norm of inputs, which steer the origin to a state $\zeta$ in a finite interval of time $[0, \Gamma]$ is $\rho$. That the Maximum Principle holds, seen through the convex analysis optics, means that the target $\zeta$ is a support vector for the set $R_{\rho}$ of all vectors reachable under an input of norm less than or equal to $\rho$. In other words the normal cone to $R_{\rho}$ at $\zeta$ is non-trivial. In the literature vectors in the normal cone, which live in the dual space, are also called multipliers, a term that has a more general meaning.

To verify that $R_{\rho}$ has support at $\zeta$, one might hope to apply to $R_{\rho}$ and $\{\zeta\}$ the celebrated Separation Theorem for linear topological spaces, stating that, given two non-void convex sets $A$ and $B$, and assuming that $A$ has interior, they can be can be separated by a continuous linear functional if and only if $B \cap A^{i}=\phi$.

Unfortunately, this application is not possible in the infinite dimensional case because it is not true in general that $\mathrm{R}_{\rho}$ has interior.

It is very well known, and easy to show, that the set of support points $S$ is contained and is dense in the set $R_{\rho}^{\wedge}$ of target points reachable with minimum input norm $\rho$. This might suggest that some of the above targets are not support point. On the other hand the cited Separation Theorem above is indeed a sufficient condition, in view of the presiding hypothesis (one of the sets has interior), so that it leaves open the problem of determining if separation holds for all targets or if, instead, the dense subset of support points is proper.

Much attention (see [1]) has been devoted to more sophisticated Banach space settings, obtaining a generalization of the Maximum principle, thanks to the definition of a larger linear space of multipliers, which contains the dual space. Similarly, this leaves to determine which multipliers are in the dual space, although sufficient conditions are known.

For details as well as for accurate historical remarks and proper credits, reference can be made to the vast and outstanding work by Fattorini, which covers a variety of Banach space settings. Recent work is, besides [1], [6] and [7].

The purpose here is to answer for Hilbert spaces the question connected to the aforementioned density results: are there vectors of $R_{\rho}^{\wedge}$ at which $R_{\rho}$ has no support?

The question remained open for quite a long time. And the answer is "no". The argument lean on a (very general) result of the theory of cones and on strict convexity of Hilbert space norms. Thus generalization are possible (although beyond the present purposes), but our technique cannot pass the barrier of the requirement of strict convexity of norms.

More specifically, we will show that the tangent cone to $R_{\rho}$ at any $\zeta \in R_{\rho}^{\wedge}$ is pointed. That some vector $\zeta \in R_{\rho}^{\wedge}$ is not a support point for $R_{\rho}$ is equivalent to say that the polar cone to the tangent cone to $R_{\rho}$ at $\zeta$ is trivial. This is in turn equivalent to say that the closure of the tangent cone is the whole space. That the closure of a pointed cone be the whole space is a rather counterintuitive proposition, and in fact we prove that this is not the case, in any linear topological space. 
From this fact it follows, as an immediate consequence, that all $\zeta \in R_{\rho}^{\wedge}$ are support points for $R_{\rho}$.

No assumptions will be made on either separability or full control. More might be said in the separable case, but this is not dealt with here.

With an apology, our exposition covers some well known basic facts and complements, in order to enhance readability. A more succinct exposition would result in a choppy and difficult to follow narration.

\section{2. $\quad$ Setting}

We refer to the Cauchy problem with $u \in \mathrm{L}_{2}\left([0, \Gamma], \mathrm{H}_{1}\right)$ as given in [2]. The abstract differential equation has the form:

$$
\dot{x}(t)=A x(t)+B u(t)
$$

where the spaces $\mathrm{H}_{1}, \mathrm{H}$ are real Hilbert spaces, B is an operator (bounded linear transformation) $H_{1} \rightarrow H, A$ is the infinitesimal generator of a strongly continuous semigroup $\{T(t)\}$ on $H$ and $x(0)=\bar{x}$ is given. The equation is intended in weak sense and its unique solution is expressed by the formula of variation of constants:

$$
x(t)=T(t) \bar{x}+\int_{[0, t]} T(t-s) B u(s) d s
$$

which formally recurs also in other settings, like that of differential equations in Banach Spaces. In our specific case the integral is a Pettis integral. The case $\mathrm{H}_{1}=\mathrm{H}$ and $\mathrm{B}=\mathrm{I}$ is referred to as full control case, but here we do not make any assumption in this respect. For simplicity, dealing with norm optimal control problems, we assume $\bar{x}=0$. The general case is a variant of this and can be obtained along the lines of [1].

It is assumed that we can reach a certain target vector $\zeta \in \mathrm{H}$ at time $\Gamma>0$ or:

$$
\zeta=\int_{[0, \Gamma]} \mathrm{T}(\Gamma-\mathrm{s}) \mathrm{Bu}(\mathrm{s}) \mathrm{d} \mathrm{s}=\mathcal{L}_{\Gamma} \mathfrak{u}
$$

and we look for the minimum norm input that does the job of reaching $\zeta$. The linear transformation $\mathcal{L}_{\Gamma}: \mathrm{L}_{2}\left([0, \Gamma], \mathrm{H}_{1}\right) \rightarrow \mathrm{H}$ is well known to be continuous. This is all we will need in the sequel as to the properties of this operator.

If the control steering the system from the origin at time 0 to $\zeta$ at time $\Gamma$ is unique, because the operator $\mathcal{L}_{\Gamma}$ is one to one, then, according to Fattorini, who introduced this concept in sixties, the system is called rigid. Fattorini constructed an example of rigid system showing that this phenomenon can actually occur (see e.g. [1] and references therein). In this case, characterizing the optimum control, becomes obviously pointless. We note that our arguments hold good even when $\mathcal{L}_{\Gamma}$ is one to one. In this case though, exploiting the fact that $\zeta$ is a support point, can only lead to retrieving the unique control that solves the reachability problem. 
It is immediate to show that the minimum norm control exists and is unique, since this is a straightforward consequence of the Projection Theorem.

\section{Lemmata and Definitions}

The environment is, unless otherwise stated, a real Hilbert space $\mathrm{H}$. The symbols $\mathcal{L}(\mathrm{C})$ and $\mathcal{L}^{-}(\mathrm{C})$ denote, respectively, the linear and closed linear hull of the non-void set $\mathrm{C}$, whereas $\mathcal{C}$ o(C) and $\mathcal{C O}^{-}(\mathrm{C})$ denote, respectively, its conical and closed conical hull.

Given a convex cone $\mathrm{C}$ in $\mathrm{H}$ its polar cone is the (always closed) cone:

$$
C^{p}=\{y:(y, x) \leq 0: \forall x \in C\}
$$

Definition 1. The tangent cone to $\mathrm{C}$ at $\mathrm{y} \in \mathrm{C}$ is the cone $\mathcal{C} \mathrm{o}(-\mathrm{y}+\mathrm{C})$, The normal cone to $\mathrm{C}$ at $\mathrm{y}$ is the polar of the tangent cone to $\mathrm{C}$ at $\mathrm{y}$.

Consider a convex set $C$ and $\zeta \in C$. We say that $\zeta$ is a support point for $C$ if there exists a nonzero vector $n$ (which can be taken with unit norm) such that:

$$
(\mathrm{n}, \zeta) \geq(\mathrm{n}, z), \forall z \in \mathrm{C}
$$

This is equivalent to say that $\mathrm{C}$ is contained in the closed half-space defined by the continuous linear functional $(n,$.$) :$

$$
\{y:(n, y) \leq(n, \zeta)\}
$$

whose limiting (closed) hyperplane contains $\{\zeta\}$. In still another equivalent terminology, this is equivalent to say that the tangent cone to $C$ at $\zeta$ has a non-trivial polar cone (because $n$ is in such cone).

Let $\mathrm{n}$ be an unit norm vector. The closed convex set:

$$
\{x: a \leq(n, x) \leq b\}
$$

(with $b \geq a$ ) is called a sandwich. The number $b-a$ is the thickness of the sandwich. If the thickness is zero, the sandwich is a closed hyperplane. The sandwich is symmetrical if it has the form

$$
\{x:-a \leq(n, x) \leq a\}
$$

for some $a \geq 0$.

The next Lemma regards closed cones in Hilbert spaces

Lemma 2. A closed cone in a Hilbert space $\mathrm{H}$ is proper if and only if it is contained in a closed half-space.

Demostración. Let C be a closed proper cone. Then there is a singleton $\{\mathrm{y}\}$ disjoint from C. Singletons are convex and compact and therefore the Strong Separation Corollary 14.4 in [4] applies. The rest is immediate. 
We recall briefly some other relevant notions about cones in infinite dimensional Hilbert Spaces. Incidentally, the literature on this topic tend to be either finite dimensional or infinite dimensional, but typically on the footsteps of the seminal work of Choquet, aiming at extending the Krein Milman Theorem in a measure theory setting.

Definition 3. A (convex) cone $\mathrm{C}$ is pointed if $\mathrm{C} \cap-\mathrm{C}=\{0\}$. A cone $\mathrm{C}$ is blunt if $\mathcal{L}^{-}(\mathrm{C})=\mathrm{H}$

The following Lemma well known.

Lemma 4. If a closed $\mathrm{C}$ cone is pointed, its polar cone is blunt, that is, $\mathcal{L}^{-}\left(\mathrm{C}^{p}\right)=\mathrm{H}$.

Demostración. The proof is simple and based on elementary computations that, for brevity, are taken for granted here, but, on the other hand, are rather intuitive, since polarization is the analogous for cones of orthogonal complementation for subspaces. We can write:

$$
\{0\}=C \cap-C=\left[C^{p}+\left(-C^{p}\right)\right]^{p}=\mathcal{L}\left(C^{p}\right)^{p}
$$

and, taking polars of the first and last cone the desired conclusion follows.

If a pointed cone is not closed what can we say of its polar cone? Notice that according to Lemma 2, if the closure of the cone is proper, then the cone has a nontrivial polar cone. For the polar to be trivial instead, again in view of the same Lemma, the closure of the cone must be the whole space, despite the fact that the cone is pointed. But we prove here that this cannot happen even in general, as stated by the following:

Theorem 5. The closure of a pointed cone in a linear topological space is a proper cone.

Demostración. Suppose that it is not true, that is there is a pointed cone $\mathrm{C}$ in a linear topological space $E$, such that $C^{-}=E$. Consider a finite dimensional subspace $F$, which intersect $C$ in a non trivial, necessarily pointed, cone. Actually we can take instead of $F$, its subspace $\mathcal{L}(F \cap C)$, without restriction of generality. For simplicity we leave the symbol $F$ unchanged, and equip $F$ with the relative topology. Next notice that, as is well known, because $F$ is the finite dimensional, the pointed convex cone $\Upsilon=\mathrm{F} \cap \mathrm{C}$ has interior. Thus it can be separated by a continuous linear functional from the origin and therefore it is contained in a closed semi-space. It follows that the closure of $\Upsilon$ in $F$ is contained in a closed half-space and therefore is a proper cone. But by Theorem 1.16 in [5], such closure is $C^{-} \cap F$. By the initial assumption $C^{-} \cap F=E \cap F=F$. This is a contradiction and therefore the proof is finished.

\section{Existence and uniqueness in $\mathrm{L}_{2}\left(\mathrm{H}_{1}\right)$}

Assume that for some $\zeta \in \mathrm{H}, \exists \mathrm{u}_{\zeta}$ such that $x=\zeta=\mathcal{L}_{\Gamma}\left(\mathfrak{u}_{\zeta}\right)$. The set of all $u$ satisfying $\zeta=\mathcal{L}_{\Gamma}(u)$ is given by $u_{\zeta}+\mathcal{N}\left(\mathcal{L}_{\Gamma}\right)$. This is a closed affine space, because $\mathcal{L}_{\Gamma}$ is continuous. If $\mathcal{N}\left(\mathcal{L}_{\Gamma}\right)$ is trivial, the unique $\mathfrak{u}_{\zeta}$ solving $\zeta=\mathcal{L}_{\Gamma}\left(\mathfrak{u}_{\zeta}\right)$ is already optimum. Optimization in this case 
is pointless, but the arguments below hold good anyway. To obtain the minimum norm solution we can apply the projection theorem and project the origin on this closed convex set. Moreover, we know, from the celebrated Projection Theorem for Hilbert spaces, that this projection exists and is unique and hence the minimum norm solution always exists and is unique. We call this unique minimum norm control $\mathrm{u}_{\mathrm{o}}$.

We can put:

$$
\left\|u_{\mathrm{o}}\right\|_{\mathrm{L}_{2}\left(\mathrm{H}_{1}\right)}=\rho
$$

which is the optimum value of the norm. In particular we can say that the optimum control belongs to the closed sphere $S_{\rho}$ of radius $\rho$, around the origin in $\mathrm{L}_{2}\left([0, \Gamma], \mathrm{H}_{1}\right)$ (briefly $\left.\mathrm{L}_{2}\left(\mathrm{H}_{1}\right)\right)$ :

$$
u_{\mathrm{o}} \in \mathrm{S}_{\rho}
$$

and so:

$$
\zeta \in \mathcal{L}_{\Gamma}\left(\mathrm{S}_{\rho}\right) \subset \mathcal{R}\left(\mathcal{L}_{\Gamma}\right)
$$

It is immediate to verify that:

$$
\left.\mathcal{L}_{\Gamma}\left(S_{\rho}\right)=\left\{z: \operatorname{mín}\left\{\|u\|: \mathcal{L}_{\Gamma}(u)=z\right\} \leq \rho\right\}\right\}
$$

For notational simplicity we put $\mathcal{L}_{\Gamma}\left(\mathrm{S}_{\rho}\right)=\mathrm{R}_{\rho}$.

\section{Reachability}

In this section we recast a few well known facts of reachability theory.

We noted that $\zeta \in \mathcal{L}_{\Gamma}\left(\mathrm{L}_{2}\left(\mathrm{H}_{1}\right)\right)=\mathcal{R}\left(\mathcal{L}_{\Gamma}\right)=\mathrm{R}_{\Gamma}$. This is the reachable set at time $\Gamma$ and is a linear subspace of $\mathrm{H}$, which is in general not closed. However, we can always argue in the Hilbert space $\mathcal{L}_{\Gamma}\left(\mathrm{L}_{2}\left(\mathrm{H}_{1}\right)\right)^{-}$and thus assume, without restriction of generality, that $\mathrm{R}_{\Gamma}$ is a dense linear subspace in $\mathrm{H}$. This is equivalent to $\mathcal{N}\left(\mathcal{L}_{\Gamma}^{*}\right)=\{0\}$. To streamline the exposition this assumption will be in force thoroughly.

Note that $\mathcal{L}_{\Gamma}^{*}$ is the map $x \rightarrow \mathrm{B}^{*} \mathrm{~T}^{*}(\Gamma-.) \mathrm{x}$. Thus $\mathrm{x} \in \mathcal{N}\left(\mathcal{L}_{\Gamma}^{*}\right)$ if and only if the continuous function $\mathrm{B}^{*} \mathrm{~T}^{*}(\Gamma-.) \mathrm{x}$ is identically zero. Next notice that:

$$
\mathcal{N}\left(\mathcal{L}_{\Gamma}^{*}\right)=\mathcal{N}\left(\mathcal{L}_{\Gamma} \mathcal{L}_{\Gamma}^{*}\right)
$$

for $\mathcal{N}\left(\mathcal{L}_{\Gamma}^{*}\right) \subset \mathcal{N}\left(\mathcal{L}_{\Gamma} \mathcal{L}_{\Gamma}^{*}\right)$ is obvious and, on the other end:

$$
\mathcal{L}_{\Gamma} \mathcal{L}_{\Gamma}^{*} x=0 \Rightarrow\left(x, \mathcal{L}_{\Gamma} \mathcal{L}_{\Gamma}^{*} x\right)=\left\|\mathcal{L}_{\Gamma}^{*} x\right\|^{2}=0
$$

Therefore, under the present hypothesis that $\mathcal{N}\left(\mathcal{L}_{\Gamma}^{*}\right)^{\perp}=\mathrm{R}_{\Gamma}^{-}=\mathrm{H}$, the selfadjoint operator

$$
\mathrm{G}_{\Gamma}=\mathcal{L}_{\Gamma} \mathcal{L}_{\Gamma}^{*}
$$


has a trivial kernel, and so it is one to one.

Moreover, obviously $\mathcal{R}\left(\mathrm{G}_{\Gamma}\right) \subset \mathcal{R}\left(\mathcal{L}_{\Gamma}\right)=\mathrm{R}_{\Gamma}$.

Indeed, $\mathcal{R}\left(\mathrm{G}_{\Gamma}\right)^{-}=\mathrm{R}_{\Gamma}^{-}$, for:

$$
\mathcal{R}\left(\mathrm{G}_{\Gamma}\right)^{-}=\mathcal{N}\left(\mathrm{G}_{\Gamma}\right)^{\perp}=\mathcal{N}\left(\mathcal{L}_{\Gamma}^{*}\right)^{\perp}=\mathrm{R}_{\Gamma}^{-}
$$

Thus both $\mathcal{R}\left(\mathrm{G}_{\Gamma}\right)$ and $\mathcal{R}\left(\mathcal{L}_{\Gamma}\right)$ are dense, so that for any $x \in \mathcal{R}\left(\mathrm{G}_{\Gamma}\right)$ and $\varepsilon>0$ there is an $y \in \mathcal{R}\left(\mathcal{L}_{\Gamma}\right)$ such that $\|x-y\| \leq \varepsilon$ and vice-versa.

Notice that, by a change of variables, $\forall x \in \mathrm{H}$ :

$$
\mathrm{G}_{\Gamma} \mathrm{x}=\int_{0}^{\Gamma} \mathrm{T}(\sigma) \mathrm{BB}^{*} \mathrm{~T}^{*}(\sigma) x \mathrm{~d} \sigma
$$

We claim that we can define the integral as a Riemann integral. In fact we can prove that the integrand is a continuous function. To this purpose first note that the function $\mathrm{BB}^{*} \mathrm{~T}^{*}(\sigma) \mathrm{x}$ is continuous. Then, to show that the integrand function is continuous, apply the exponential growth property of the semigroup and following well known:

Theorem 6. Consider a set $\mathcal{T} \subset \mathrm{LC}(\mathrm{H}, \mathrm{H})$ and suppose that it is bounded in the norm operator topology. Then the evaluation map is jointly continuous in $\mathcal{T} \times \mathrm{H}$ where $\mathcal{T}$ is equipped with the (relativized) pointwise topology.

If the state $\mathrm{y}$ is reachable and $\mathrm{y} \in \mathcal{R}\left(\mathrm{G}_{\Gamma}\right)$,

$$
y=G_{\Gamma} x
$$

has solution in $\mathrm{x}$, so that

$$
y=\int_{0}^{\Gamma} \mathrm{T}(\sigma) \mathrm{BB}^{*} T^{*}(\sigma) \mathrm{d} \sigma x=\int_{0}^{\Gamma} \mathrm{T}(\Gamma-\tau) \mathrm{B} w(\tau) \mathrm{d} \tau
$$

where $w(\tau)=\mathrm{B}^{*} \mathrm{~T}^{*}(\Gamma-\tau) x$. In this case, there is a smooth control that solves the problem.

\section{The Quasi-Topology of $R_{\rho}$ and Main Theorem}

There are a number of interesting properties of $R_{\rho}=\mathcal{L}_{\mathrm{T}}\left(S_{\rho}\right)$, which depend both on the environment (Hilbert space), on continuity of $\mathcal{L}_{\mathrm{T}}$ and on the fact that the closed sphere $\mathrm{S}_{\rho}$ is convex and weakly compact. In particular recall that $s-\mathrm{s}$ (strong-strong) continuity is equivalent to $w-w$ (weak-weak) continuity and, therefore, $\mathcal{L}_{\mathrm{T}}$ is $w-w$ continuous.

The set $\mathrm{R}_{\rho}$ is obviously: 
- Convex (as image under an operator of a convex set) with $0 \in R_{\rho}$, and symmetrical.

- Weakly compact, as image of a weakly compact set under a $w-w$ continuous linear transformation

- Weakly closed + convex and hence strongly closed

- Bounded (as image under an operator of a bounded set)

- Not a convex body in general.

We now describe what we mean with the quasi-topology of $\mathrm{R}_{\rho}$, adding to the above list the fact that $\mathrm{R}_{\rho}$ has a "quasi-interior", at whose points it is densely radial and circled, and a quasiboundary, which is the complement in $\mathrm{R}_{\rho}$ of the quasi-interior. These terms are justified because this quasi-topology can be realized as an actual topology, using the topology introduced by Fattorini for $\mathrm{R}_{\rho}$, based on the norm:

$$
p(z)=\inf \left\{\|u\|: \mathcal{L}_{\Gamma} u=z\right\}
$$

This concept is of primary importance in more general and complex Banach space settings, but it will not be used here.

Definition 7 . The set of all vectors $x$ in $R_{\rho}$, such that the minimum norm control to reach $x$ has norm $\rho$, is called quasi-boundary of $R_{\rho}$ and denoted by $R_{\rho}^{\wedge}$. The set of all vectors $x$ in $R_{\rho}$, such that the minimum norm control to reach $x$ has norm $\rho^{\prime}<\rho$, is called quasi-interior of $R_{\rho}$ and denoted by $R_{\rho}^{\vee}$.

It is well known that no point of the quasi-interior can be a support point for $\mathrm{R}_{\rho}$.

Consider the origin, which belongs to $\mathrm{R}_{\rho}^{\vee}$. The set $\mathrm{R}_{\rho}$ is densely radial at the origin. In fact consider any point $z \in R_{\Gamma}$ and let $\eta$ be the minimum norm of the unique control $u$ that reaches $z$. If $\eta \leq \rho$ the whole segment $[0: z] \subset \mathcal{L}_{\Gamma}\left(S_{\rho}\right)$. Otherwise take the point $z^{\prime}$ reached by the control $\frac{\rho}{\eta} \mathrm{u}$, and $\left[0: z^{\prime}\right] \subset \mathcal{L}_{\Gamma}\left(S_{\rho}\right)$. Observe that $\forall z \in R_{\Gamma}$ is positively proportional to a vector in $\mathcal{L}_{\Gamma}\left(S_{\rho}\right)$. In other words:

$$
\cup\left\{\alpha \mathcal{L}_{\Gamma}\left(S_{\rho}\right): \alpha>0\right\}=R_{\Gamma}
$$

Moreover it is clear that $\alpha \mathcal{L}_{\Gamma}\left(S_{\rho}\right) \subset \mathcal{L}_{\Gamma}\left(S_{\rho}\right)$ for any positive $\alpha \leq 1$, so that $\mathcal{L}_{\Gamma}\left(S_{\rho}\right)$ is also circled.

On the other hand, if for $\zeta \in \mathcal{L}_{\Gamma}\left(S_{\rho}\right)$ the minimum norm of the corresponding control to reach $\zeta$ is $\rho$, by a very well known argument, see e.g. [1], $(1+\varepsilon) \zeta \notin \mathcal{L}_{\Gamma}\left(S_{\rho}\right)$ for $\forall \varepsilon>0$.

All the points of the quasi-interior have the same dense radiality property as the origin. In fact, if $\xi \in R_{\rho}^{\vee}, \xi \neq 0$, then the minimum norm of the control that steers the origin to the state to $\xi$ is some $\rho^{\prime}$ with $0<\rho^{\prime}<\rho$. Let $\mathfrak{u}_{\xi}$ be the corresponding minimum norm control. Radiality in the direction of the origin or of $\xi$ itself is obvious. Next consider any $z \in R_{\Gamma}$ with $z \neq 0$ and $z$ not proportional to $\xi$, and let $\mathrm{u}_{z}$ be corresponding the minimum norm control. It will be $\left\|\mathrm{u}_{z}\right\|=\gamma>0$. Then it is immediate that $\xi+\alpha z \in \mathrm{R}_{\rho}$ for any $\alpha$, such that: $0<\alpha \leq \frac{\rho-\rho^{\prime}}{\gamma}$.

The role of the dense radiality is emphasized by the proof of the anticipated well known result: 
Theorem 8. No point of $R_{\rho}^{\vee}$ can be a support point of $R_{\rho}$.

Demostración. Suppose that a point $y \in R_{\rho}^{\vee}$ is a support point. Then the projection of some vector $z \notin R_{\rho}$ on $R_{\rho}$ is $y$. By the Projection Theorem we have:

$$
(z-y, x-y) \leq 0, \forall x \in R_{\rho}
$$

If equality holds for all $x \in R_{\rho}$ then $R_{\rho}$ and hence also $R_{\Gamma}$ would be contained in a closed hyperplane, contradicting that $R_{\Gamma}$ is dense in the space. So $\exists x \in R_{\rho}$ such that $(z-y, x-y)<0$. Because of the radiality property at $y$ we can take in lieu of $x$, a state $w=y+\alpha(y-x) \in R_{\rho}$ for some $\alpha>0$. But then $(z-y, w-y)=\alpha(z-y, y-x)>0$ contradicting the Projection Theorem.

At this point we know that any support point is in $\mathrm{R}_{\rho}^{\wedge}$.

It is well known that support points are dense in the quasi-boundary of $\mathrm{R}_{\rho}($ e.g. [2]).

To show this, one may use the following sequence of support points converging to an arbitrary $\zeta \in \mathrm{R}_{\rho}^{\wedge}$. For any positive integer $\mathrm{i},\left(1+\frac{1}{\mathrm{i}}\right) \zeta \notin \mathrm{R}_{\rho}$. Because the projection $\mathrm{P}_{\mathrm{R}_{\rho}}$ on $\mathrm{R}_{\rho}$ is continuous, the sequence $\left\{\mathrm{P}_{\mathrm{R}_{\rho}}\left(\left(1+\frac{1}{i}\right) \zeta\right)\right\}$ converges strongly to $\zeta$ and obviously, because the points in the sequence are projections, they are all support points (and hence also lie on the quasi-boundary of $\left.R_{\rho}\right)$.

However, more is true for arbitrary vectors of the quasi-boundary of $\mathrm{R}_{\rho}$ in the present Hilbert space setting, as we show in the next:

Theorem 9. All vectors in $\mathrm{R}_{\rho}^{\wedge}$ are extreme. No other point of $\mathrm{R}_{\rho}$ can be extreme

Demostración. Suppose that for the quasi-boundary vector $\zeta$ it is true that $\zeta=\frac{\zeta_{1}+\zeta_{2}}{2}$ with $\zeta_{1}$ and $\zeta_{2}$ in $R_{\rho}$, and let $\rho_{1} \leq \rho$ be the norm of the minimum norm control $u_{1}$ that steers the system to $\zeta_{1}$ and $\rho_{2} \leq \rho$ be the norm of the minimum norm control $u_{2}$ that steers the system to $\zeta_{2}$. The control $\frac{\mathfrak{u}_{1}+\mathfrak{u}_{2}}{2}$ has norm strictly less than $\rho$, because the norm of a Hilbert space is strictly convex, and steers the system to $\zeta$. But this contradicts that $\zeta \in \mathrm{R}_{\rho}^{\wedge}$, and so the proof of the first statement is finished. Points of the quasi-interior cannot be extreme in view of the specific dense radiality property we have illustrated. Therefore we are done.

Corollary 10. The tangent cone to $\mathrm{R}_{\rho}$ at any $\zeta \in \mathrm{R}_{\rho}^{\wedge}$ is pointed

Demostración. Obviously if it were not so it would be contradicted that $\zeta$ is an extreme point.

At this point, putting together this Corollary with Lemmata 5 and 2 we have established the following main

Theorem 11. Any point $\zeta \in \mathrm{R}_{\rho}^{\wedge}$ is a support point of $\mathrm{R}_{\rho}$. In other words

$$
\forall \zeta \in \mathrm{R}_{\rho}^{\wedge}, \exists \mathrm{n} \in \mathrm{H}^{*}=\mathrm{H},\|\mathrm{n}\|=1
$$


such that

$$
(\mathrm{n}, \zeta) \geq(\mathrm{n}, z), \forall z \in \mathrm{C}
$$

Given this result we may expect all the more a large normal fan for $\mathrm{R}_{\rho}$. Indeed the normal fan is the whole space, but this fact is independent on the main Theorem, as we illustrate in the next section.

\section{The Set of Support Points and the Normal Fan}

In this Section we collect some, mostly well known, facts about the set of support points $\mathrm{S}$ (which we now know to be the same as $\mathrm{R}_{\rho}^{\wedge}$ ) and the normal fan. At each support point $\zeta$ the tangent cone has a non-trivial polar cone, also called the normal cone at $\zeta$. The union of these cones is the normal fan of $\mathrm{R}_{\rho}$. Naturally, it is often convenient to normalize vectors in the normal fan.

Suppose $\zeta$ is a support point of $\mathrm{R}_{\rho}$. Using the pairing in $\mathrm{H}$ and the CBS inequality, we have a well known expression for $\zeta$. Let $\mathrm{n}$ a unit norm vector in the normal cone at $\zeta$. Then it must be for any $\mathrm{y} \in \mathrm{R}_{\rho}$ :

$$
(\mathrm{n}, \mathrm{y}-\zeta)=\left(\mathrm{n}, \mathcal{L}_{\Gamma} \mathrm{u}_{\mathrm{y}}-\mathcal{L}_{\Gamma} \mathrm{u}_{\zeta}\right) \leq 0
$$

or

$$
\left(\mathrm{n}, \mathcal{L}_{\Gamma} \mathrm{u}_{\mathrm{y}}\right) \leq\left(\mathrm{n}, \mathcal{L}_{\Gamma} \mathrm{u}_{\zeta}\right)
$$

or

$$
\left(\mathcal{L}_{\Gamma}^{*} n, u_{y}\right) \leq\left(\mathcal{L}_{\Gamma}^{*} n, u_{\zeta}\right)
$$

so that, by the CBS inequality, the optimal control corresponding to $\zeta$ has the expression:

$$
u_{\zeta}=\rho \frac{\mathcal{L}_{\Gamma}^{*} n}{\left\|\mathcal{L}_{\Gamma}^{*} n\right\|}, \text { a.e. }
$$

and:

$$
\zeta=\mathcal{L}_{\Gamma} \frac{\rho}{\left\|\mathcal{L}_{\Gamma}^{*} n\right\|} \mathcal{L}_{\Gamma}^{*} n=\frac{\rho}{\left\|\mathcal{L}_{\Gamma}^{*} n\right\|} G_{\Gamma} n
$$

On the other hand, for an arbitrary unit norm vector $n$ consider the linear program:

$$
\operatorname{máx}\left\{(n, z): z \in R_{\rho}\right\}
$$

The existence of solutions of this linear program derive from the fact that $\mathrm{R}_{\rho}$ is convex and weakly compact and the functional is continuous (and hence also weakly continuous). Let $\zeta$ be a solution, then for any $\mathrm{y} \in \mathrm{R}_{\rho}$ :

$$
(n, y-\zeta) \leq 0
$$

so that $\zeta$ is a support point and has the above expression. 
Thus

$$
\mathrm{S}=\left\{\zeta: \zeta=\frac{\rho}{\left\|\mathcal{L}_{\Gamma}^{*} \mathrm{n}\right\|} \mathrm{G}_{\Gamma} \mathrm{n},\|\mathrm{n}\|=1\right\}
$$

Notice that because $\mathrm{G}_{\Gamma}$ is one-to-one, to each support $\zeta$ there corresponds a unique normal $\mathrm{n}$. In other words, all normal cones are rays. We collect these facts and more in the following:

Theorem 12. The normal fan of $R_{\rho}$, less the origin, is the whole space $H \backslash\{0\}$. All normal cones at support vectors $\zeta$ are rays, under the correspondence between unit normal vectors $n$ and support points $\zeta$ :

$$
\zeta=\frac{\rho}{\left\|\mathcal{L}_{\Gamma}^{*} \mathrm{n}\right\|} \mathrm{G}_{\Gamma} \mathrm{n}
$$

The correspondence between unit vectors in the normal fan and the corresponding support points is one-to-one. If $\zeta$ is a support point $\{\zeta\}$ is an exposed face of $R_{\rho}$. Equivalently, for any pair normal-support point it is true that:

$$
(\mathrm{n}, \mathrm{y}-\zeta)<0, \forall \mathrm{y} \in \mathrm{R}_{\rho}
$$

Consequently ( since $0 \in R_{\rho}$ ) the value of the functional $(n,$.$) at \zeta$ is always positive.

Demostración. It remains to be proved the last statement. Suppose a $\zeta \in R_{\rho}$ is a support point, so that it is necessarily a quasi-boundary and extreme point. Suppose that for some normal $n$ there is $z$ such that:

$$
(n, z-\zeta)=0
$$

then $z$ is a support point as well with normal $n$. In fact, $\forall y \in R_{\rho}$ :

$$
(n, y-z)=(n, y-\zeta)+(n, \zeta-z) \leq 0
$$

Therefore $z$ is a support point and hence it is an quasi-boundary point, so that the minimum norm control that steers the system to $z$ has norm $\rho$. Denote by $u_{z}$ the minimum norm control to reach $z$ and by $u_{\zeta}$ the minimum norm control to reach $\zeta$. By the same argument above all points in $[\zeta: z]$ are support points with normal $n$. In fact for $0<\alpha<1, \forall y \in R_{\rho}$ :

$$
(n, y-(\zeta+\alpha(z-\zeta))) \leq 0
$$

But the point $\frac{\zeta+z}{2}$ can be reached by the control $\frac{\mathfrak{u}_{\zeta}+\mathfrak{u}_{z}}{2}$ and $\left\|\frac{\mathfrak{u}_{\zeta}+\mathfrak{u}_{z}}{2}\right\|<\rho$. This means that $\frac{\zeta+z}{2}$ is a quasi-interior point, which is a contradiction and the proof is finished.

Returning to the linear program:

$$
\operatorname{máx}\left\{(n, z): z \in R_{\rho}\right\}
$$

Call the maximum $\mathrm{m}$, then

$$
m=\frac{\rho}{\left\|\mathcal{L}_{\Gamma}^{*} n\right\|}\left(n, G_{\Gamma} n\right)=\rho\left(n, G_{\Gamma} n\right)^{1 / 2}=\rho\left\|\mathcal{L}_{\Gamma}^{*} n\right\|^{1 / 2}
$$


Recall that $\mathrm{m}>0$. Because $\mathrm{R}_{\rho}$ is symmetric, it is contained in the symmetrical sandwich:

$$
R_{\rho} \subset\{y:-m \leq(n, y) \leq m\}
$$

and the limiting hyperplanes of the sandwich meet $R_{\rho}$ only in the two points $\zeta$ and $-\zeta$. Also the sandwich cannot degenerate to a hyperplane, because its thickness is $2 \mathrm{~m}>0$.

Received: October 2009. Revised: January 2010.

\section{Referencias}

[1] H.O. Fattorini, Infinite Dimensional Linear Control Systems, Elsevier, Amsterdam 2005.

[2] A.V. Balakrishnan, Applied Functional Analysis,Springer-Verlag Berlin-Heidelberg-New York 1976 .

[3] P.R. Halmos, A Hilbert Space Problem Book Van Nostrand, New York, 1967.

[4] J.L. Kelley and I. Namioka, Linear Topological Spaces, Springer, New York, 1963

[5] J.L. Kelley, General Topology, Springer, New York, 1955.

[6] H.O. Fatrorini Smoothness of the Costate and the Target in the Time and Norm Optimal Problems Optimization, Vol.55, No.2, 2006, 19-36

[7] H.O. Fattorini Regular and Strongly Regular Time and Norm Optimal Controls to appear 